Article

\title{
Equivalent Linearization Methods for a Control System with Clutching Inerter Damper
}

\author{
Luyu Li ${ }^{1,2}$, Qigang Liang ${ }^{1, *(1)}$ and Han Qin ${ }^{1}$ \\ 1 School of Civil Engineering, Dalian University of Technology, Dalian 116024, China; \\ liluyu@dlut.edu.cn (L.L.); tsinhan@mail.dlut.edu.cn (H.Q.) \\ 2 State Key Laboratory of Coastal and Offshore Engineering, Dalian University of Technology, \\ Dalian 116024, China \\ * Correspondence: liangqigang@mail.dlut.edu.cn
}

Received: 25 January 2019; Accepted: 12 February 2019; Published: 17 February 2019

Featured Application: This work aims to linearize the CID system, which has inherent nonlinearity, in preparation for further performance evaluation and design for structural vibration control with a CID.

\begin{abstract}
Inerter-based dampers have gained great popularity in structural vibration control. In this paper, equivalent linearization methods (ELMs) for a single-degree-of-freedom (SDOF) system with a clutching inerter damper (CID) are studied. The comparison of a SDOF system with a CID and an inertial mass damper (IMD) shows the advantage of the CID. Considering that the system with the CID is nonlinear, which is problematic for its performance evaluation and the integrated design of the structure and control system, three equivalent linearization methods based on different principles are proposed and discussed in this paper. The CID is considered to be equal to a combination of an IMD and a viscous damper. The equivalent inertance and damping can be calculated using the obtained formulas for all methods. In addition, all methods are compared in a numerical study. Results show that the ELM based on period and energy is recommended for small inertance-mass ratios.
\end{abstract}

Keywords: inertial mass damper; clutching inerter damper; equivalent linearization method; statistical linearization method; performance evaluation

\section{Introduction}

Recently, the structural vibration control strategy has been widely applied in civil engineering [1-6]. New passive control devices, such as shape memory alloy dampers [7,8], pounding dampers [9,10], and magnetorheological dampers [11,12], can suppress structural vibration effectively. As an innovative control device, the inertial mass damper (IMD) has gained great popularity in the field of structural vibration control. Smith [13] proposed the concept of inerter, which was first applied in vehicle suspension systems [14]. The mechanical model of the IMD involves an element with two nodes, and the force of the element is positively correlated with the relative acceleration of the two nodes. A study showed that the inerter achieves a damping effect by reducing the load intensity [15], and extends the period of the structure without reducing its stiffness, which is different from base isolation.

Many inerter-based dampers have been proposed and studied in the field of civil engineering. One possible problem in structures equipped with an IMD is that the IMD or its support may bear a huge force after the structure attains its peak velocity and begins to slow down. This is because of the deceleration delay of the IMD, which results in a faster movement of the damper than the structure. Subsequently, the IMD pushes the structure to move, and the IMD and its support bear 
a huge force in the meantime, which may be destructive for the system. Taking this problem into account, an IMD with a clutch, known as clutching inerter damper (CID), was proposed $[15,16]$. The CID works like a bicycle ratchet. In other words, it works when the velocity and acceleration move in the same direction and does not work when they move in the opposite direction. Hence, the CID can reduce the displacement response more effectively after the point when the structure starts to slow down. However, the effectiveness of the CID has not been evaluated clearly in previous studies. Although the working mechanism of the CID is clear, it is difficult for designers to apply it in structures without certain linearization methods, since the equation of the CID is nonlinear, compared with the traditional IMD.

For a structure with a CID, the time-history analysis can be used for numerical analysis. However, considering the integrated design of structure and CID, it is better to linearize the equation. Therefore, an equivalent linearization method (ELM) needs to be developed. For systems with a bilinear hysteresis model, there have been numerous conventional equivalent linearization methods [17-20]. For other nonlinear models, the statistical linearization method is widely used [21-24]. Several new linearization methods were also proposed in recent years [25-28]. The linearization method for the CID based on the equivalent period and energy was discussed by Wang et al. [16].

In this paper, the equation of motion of a single-degree-of-freedom (SDOF) system with a traditional IMD is obtained by theoretical analysis; subsequently, the responses of the SDOF system with an IMD and a CID under sinusoidal excitation are compared using numerical simulation. Secondly, three equivalent linearization methods are proposed. The first ELM is based on the adaptation of the frequency response function (FRF), which is denoted as ELM1. The second ELM is based on the equivalent period and the conservation of energy, denoted as ELM2. The third ELM is based on statistical linearization, denoted ELM3. Finally, a numerical example is provided to evaluate the accuracy of all methods under different excitations.

\section{Analysis of SDOF System with CID}

\subsection{Equation of Motion of SDOF with IMD}

In order to compare the CID with a traditional IMD, the equation of motion of a structure with a traditional IMD is derived in this section. Figure 1 shows a SDOF system with a traditional IMD based on gear and flywheel. The IMD is supported by a rigid frame, whose deformation can be ignored. The IMD, which is made up of a gear with a radius and a flywheel with a radius supported by the frame through the fixed spiale. When the structure begins to move, the translational motion of the beam drives the gear to rotate and the flywheel rotates at the same time.

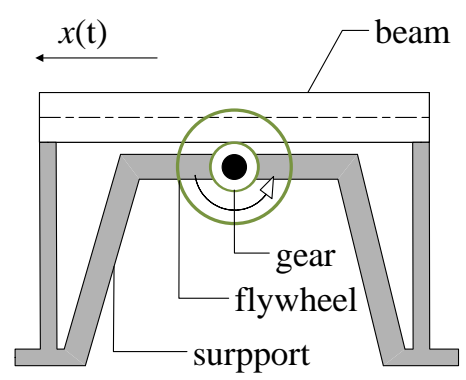

(a)



(b)

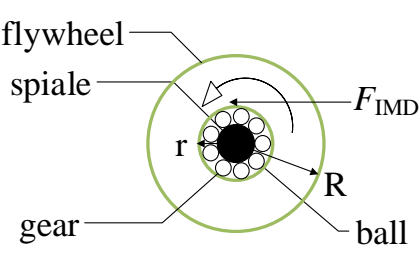

(c)

Figure 1. A single-degree-of-freedom (SDOF) system with an inertial mass damper (IMD): (a) Front view; (b) Side view; (c) Detailed view.

The equation of motion of the system under seismic load is

$$
m \ddot{x}+c \dot{x}+k x+F_{I M D}(t)=-m \ddot{x}_{g}
$$


where $m, c$, and $k$ are the mass, damping, and stiffness of the SDOF system, respectively; $x$ is the relative displacement response of the SDOF system, representatively; $\ddot{x}_{g}$ is the acceleration of ground motion; and $F_{I M D}(t)$ is the force provided by the IMD, which can be derived by the following analysis.

From Figure 1c, it is obvious that the IMD satisfies the following moment equilibrium equation:

$$
F_{I M D}(t) \cdot r=J \cdot \ddot{\theta}(t)
$$

where $J=M R^{2} / 2$ represents the moment of inertia of the IMD and M is the flywheel mass of the IMD; $\ddot{\theta}(t)$ is the angular acceleration of the IMD. Equation (2) is considered to be established ideally, ignoring the gear friction. The translational motion of the beam drives the gear to rotate. It can be obtained that

$$
\ddot{x}(t)=\ddot{\theta}(t) \cdot r
$$

Substituting Equation (2) and Equation (3) into Equation (1), $F_{I M D}(t)$ can be expressed as

$$
F_{I M D}(t)=b \ddot{x}(t)
$$

where $b=M R^{2} / 2 r^{2}$ is the inertance of the IMD, also called apparent mass by Ikago et al. [20]. Substituting Equation (4) into Equation (1), the equation of motion of the IMD system becomes

$$
(m+b) \ddot{x}+c \dot{x}+k x=-m \ddot{x}_{g}
$$

The ratio of $b$ to $m$ is known as the inertance-mass ratio, which is expressed as

$$
\mu=\frac{b}{m}
$$

Equation (5) can be normalized by dividing by $m+b$ on both sides:

$$
\ddot{x}+2 \frac{\xi}{\sqrt{1+\mu}} \frac{\omega}{\sqrt{1+\mu}} \dot{x}+\left(\frac{\omega}{\sqrt{1+\mu}}\right)^{2} x=-\frac{\ddot{x}_{g}}{1+\mu}
$$

where $\omega$ and $\xi$ are the natural circular frequency and damping ratio of the SDOF system, respectively:

$$
\omega=\sqrt{\frac{k}{m}} ; \xi=\frac{c}{2 \sqrt{k m}}
$$

\subsection{Equation of Motion of SDOF with CID}

The CID only works when the velocity and acceleration of the structure move in the same direction, working as an IMD. Figure 2 shows a SDOF system with a CID. The difference with the IMD is that the gear is added with a ratchet with a clutch effect.

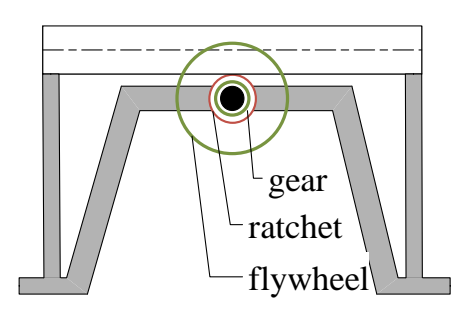

(a)

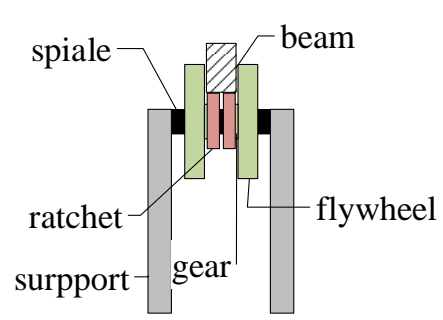

(b)

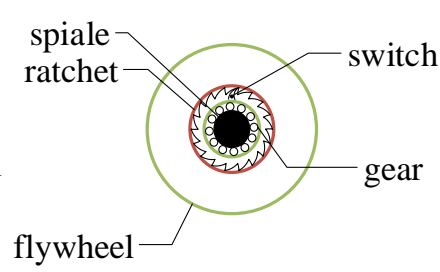

(c)

Figure 2. A SDOF system with clutching inerter damper (CID): (a) Front view; (b) Side view; (c) Detailed view. 
There are two independent flywheels in the CID, both equipped with a ratchet. Note that the two ratchets are installed in the opposite direction, in other words, only one of them works when the structure moves in one direction. The equation of motion of a SDOF system with CID [16] is

$$
\begin{aligned}
& m \ddot{x}+c \dot{x}+k x+F_{C I D}(t)=-m \ddot{x}_{g} \\
& F_{\mathrm{CID}}(t)=\left\{\begin{array}{c}
b \ddot{x}, \text { when }[\dot{x} \cdot \ddot{x}]>0 \\
0, \text { when }[\dot{x} \cdot \ddot{x}] \leq 0
\end{array}\right.
\end{aligned}
$$

where $\mathrm{b}$ is the inertance of the CID when it works, and $F_{C I D}(t)$ is the force provided by the CID. In the equation, the CID is defaulted to be at rest whenever the structure starts to move in each circle.

\subsection{Comparison of System with IMD and CID}

In this section, a comparison between the SDOF system with a CID and a IMD is performed. The damping ratio of the original SDOF system is set to 0.05 , and the frequency to $2 \pi \mathrm{rad} / \mathrm{s}$. Figure 3 shows the peak displacement response of the IMD and CID systems under sinusoidal excitation $\sin (\Omega \mathrm{t})$.

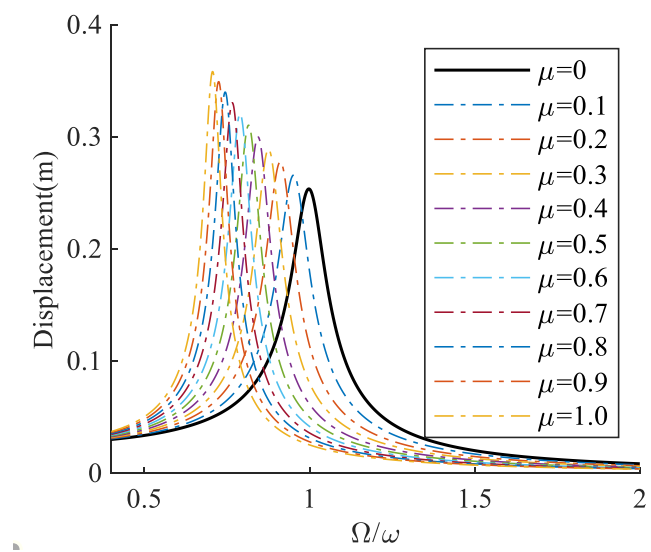

(a)

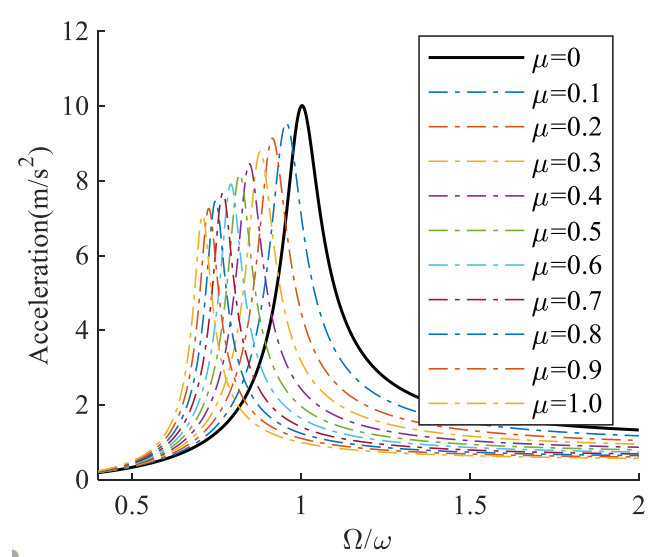

(c)

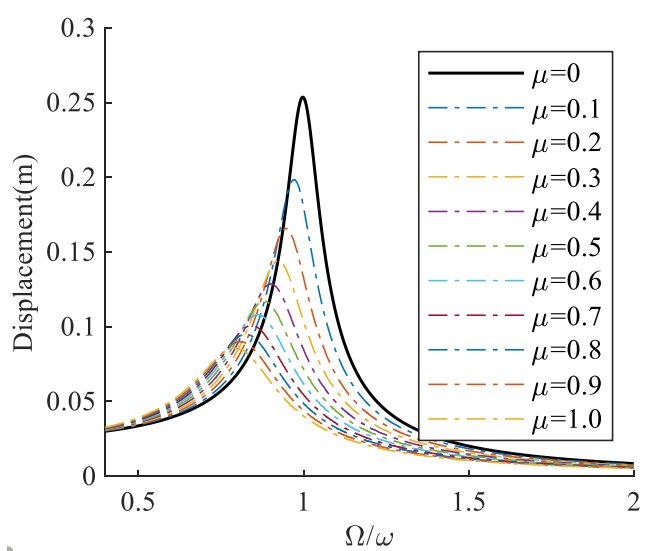

(b)

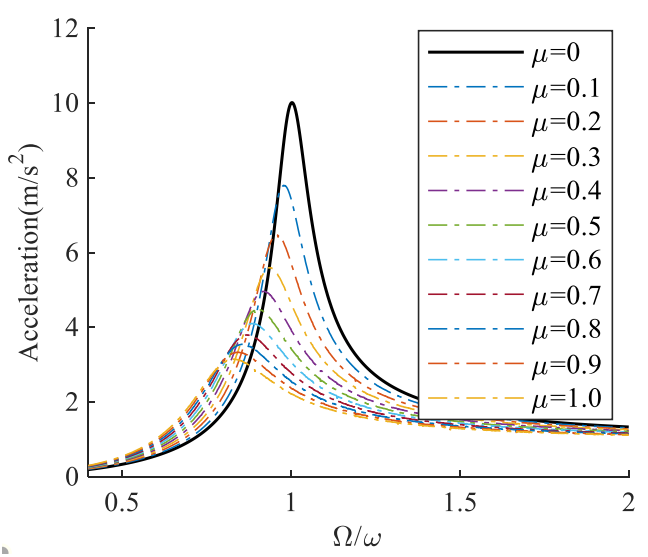

(d)

Figure 3. Steady-state peak responses for a SDOF system with IMD and CID under sinusoidal excitation. (a) Displacement of IMD system; (b) Displacement of CID system; (c) Acceleration of IMD system; (d) Acceleration of CID system.

As shown in Figure 3, for both systems, the resonance frequency is reduced as the inertance-mass ratio increases because of the supplement inertial mass. In the resonant response, the acceleration response of the IMD and CID systems can be reduced, with the latter showing a higher reduction. The displacement response of the CID system can be reduced significantly in the peak level, showing its 
superiority compared with the IMD system, which will amplify the resonant response. However, the nonlinear characteristics of the CID system determine that the responses of the CID system may only be calculated by simulation or other complicated nonlinear analysis. It remains difficult for engineers to design structures like this, although the method of time-history analysis is easy and reliable. Thus, an ELM for systems with a CID needs to be developed.

Studies have shown that the inertance-device mass ratio can reach a large value. The inertance-device mass ratio was greater than 3000 in the study of Nakamura et al. [29]. A large inertance-device mass ratio will achieve a large inertance-mass ratio $\mu$ as defined in Equation (6). Therefore, the maximum value of inertance-mass ratio $\mu$ is taken as 1 in the following linearization analysis.

\section{ELM1 Based on the Adaptation of FRF}

In this section, an ELM for a SDOF system with CID is proposed based on the adaptation of FRF of the CID system and an equivalent linear system (ELS). As shown in Figure 3, the CID can reduce not only the frequency of the structure but also its peak response. Therefore, it is reasonable to consider that the CID is equal to a combination of an IMD and a viscous damper.

The equation of motion of the ELS can be written as

$$
\left(m+b_{e q}\right) \ddot{x}+\left(c+c_{e q}\right) \dot{x}+k x=-m \ddot{x}_{g}
$$

in which $b_{e q}$ and $c_{e q}$ are the equivalent inertance and equivalent damping of the CID, respectively. The FRF of the ELS is given by

$$
H(\Omega)=\frac{m}{k-\left(m+b_{e q}\right) \Omega^{2}+i \Omega\left(c+c_{e q}\right)}
$$

The amplitude of $H(\Omega)$ written as $|H(\Omega)|$ is given by

$$
|H(\Omega)|=\frac{1}{1+\mu_{e q}} \frac{1}{\sqrt{\left(\omega_{e q}^{2}-\Omega^{2}\right)^{2}+\left(2 \xi_{e q} \Omega \omega_{e q}\right)^{2}}}
$$

where $\omega_{e q}$ and $\xi_{e q}$ are defined as

$$
\begin{gathered}
\omega_{e q}=\sqrt{\frac{k}{m+b_{e q}}}=\frac{1}{\sqrt{1+\mu_{e q}}} \omega \\
\xi_{e q}=\frac{c+c_{e q}}{2 \sqrt{k\left(m+b_{e q}\right)}}=\left(1+\frac{c_{e q}}{c}\right) \frac{1}{\sqrt{1+\mu_{e q}}} \xi
\end{gathered}
$$

According to the theory of resonance reaction, $|H(\Omega)|$ reaches the peak at the frequency

$$
\Omega=\omega_{e q} \sqrt{1-2 \xi_{e q}^{2}}
$$

Substituting Equation (16) into Equation (13), the maximum amplitude of $|H(\Omega)|$ is given by

$$
|H(\Omega)|_{\max }=\frac{1}{\omega^{2}} \frac{1}{2 \xi_{\text {eq }} \sqrt{1-\xi_{e q}^{2}}}
$$


Considering Figure 3b, the ordinate is the steady-state displacement amplitude of the CID systems with different inertance to mass ratios under sinusoidal input of unit amplitude. The steady-state reaction of the ELS under the input of $\sin (\Omega \mathrm{t})$ can be expressed as

$$
\begin{gathered}
x_{p}(\Omega, t)=|H(\Omega)| \sin (\Omega t-\theta) \\
x_{p}(\Omega, t)_{\max }=|H(\Omega)| \\
U_{\max }=|H(\Omega)|_{\max }
\end{gathered}
$$

where $x_{p}(\Omega, t)$ is the steady-state displacement response under the input of $\sin (\Omega t) ; \theta$ is the phase angle with which the response lags behind the applied loading; $x_{p}(\Omega, t)_{\max }$ is the maximum steady-state displacement response, which is equal to the amplitude of $H(\Omega)$; and $U_{\max }$ is the resonance response, which is equal to the peak of $|H(\Omega)|$.

For any $\mu$ in Figure $3 b, U_{\max }$ and its corresponding $\Omega$ is easy to find. Substituting them into Equation (16), Equation (17) and Equation (20), the equivalent linearization parameters are obtained:

$$
\begin{gathered}
\xi_{e q}=\sin \left(\frac{1}{2} \arcsin \left(\frac{1}{\omega^{2} U_{\max }}\right)\right) \\
\omega_{e q}=\frac{\Omega}{\sqrt{1-2 \xi_{\text {eq }}^{2}}} \\
\mu_{e q}=\left(\frac{\omega}{\omega_{e q}}\right)^{2}-1, \\
b_{e q}=\mu_{e q} m=\left(\left(\frac{\omega}{\omega_{e q}}\right)^{2}-1\right) m
\end{gathered}
$$

After substituting $\xi_{e q}$ and $\mu_{e q}$ into Equation (15), $c_{e q}$ is finally obtained:

$$
c_{e q}=c\left(\frac{\xi_{e q}}{\xi} \sqrt{1+\mu_{e q}}-1\right)
$$

\section{ELM2 Based on Period and Energy}

In this section, an ELM based on the equal decay period and conservation of energy in one circle of free vibration is discussed. This method was discussed by Wang et al. [16]. In this paper, the principle to specify the equivalent damping is revised and better results can be obtained.

\subsection{Equivalent Inertance}

As can be seen from Figure 4, the vibration of the structure in one cycle can be divided into four stages.

From 0 to $t_{1}$. The structure begins to move after given an initial displacement $x(0)=x_{0}$, and the CID starts working as an IMD until the structure reaches its peak velocity at time $t_{1}$. In this stage, the structure vibrates at the frequency of $\omega_{I M D}$ for a quarter of cycle. Thus, $t_{1}$ is given by

$$
t_{1}=\frac{\pi}{2 \omega_{I M D}}=\frac{\pi}{2} \sqrt{\frac{m+b}{k}}
$$



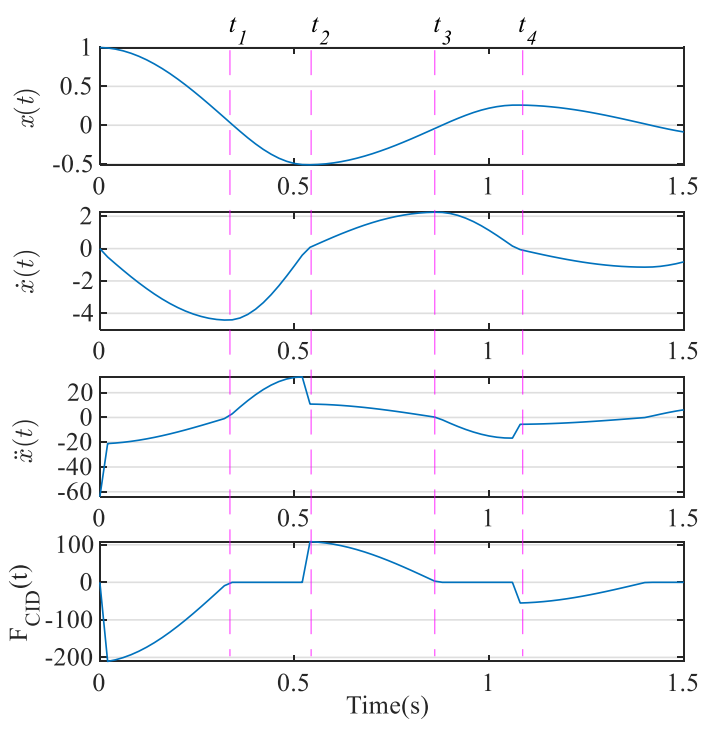

Figure 4. Responses of free vibration of SDOF system with CID $(\mu=1)$.

From $t_{1}$ to $t_{2}$. At time $t_{1}$, the structure reaches the peak velocity, the acceleration is decreased to zero in the meantime, and its direction reverses at the next moment. The speed of the structure begins to decrease and the CID does not work for the structure because of the clutch effect. During this stage, the structure vibrates at the frequency of $\omega$ for a quarter of cycle. Thus, the time elapsed in this stage is

$$
t_{2}-t_{1}=\frac{\pi}{2 \omega}=\frac{\pi}{2} \sqrt{\frac{m}{k}}
$$

From $t_{2}$ to $t_{3}$. At time $t_{2}$, the structure reaches the maximum displacement in the opposite direction to that at the initial time. Then, the other CID comes into use until time $t_{3}$. During this stage, the structure vibrates at the frequency of $\omega_{I M D}$ for a quarter of cycle. Thus, the time elapsed in this stage is

$$
t_{3}-t_{2}=\frac{\pi}{2 \omega_{I M D}}=\frac{\pi}{2} \sqrt{\frac{m+b}{k}}
$$

From $t_{3}$ to $t_{4}$. At time, the structure reaches the maximum velocity in the opposite direction to that at time $t_{1}$. Then, the other CID is divorced from structure. In this stage, the structure vibrates at the frequency of $\omega$ for the last quarter of cycle. Thus, the time elapsed in this stage is

$$
t_{4}-t_{3}=\frac{\pi}{2 \omega}=\frac{\pi}{2} \sqrt{\frac{m}{k}}
$$

The total time during one circle can be determined by summing the four time stages above:

$$
T_{C I D}=\frac{\pi}{\omega}+\frac{\pi}{\omega_{I M D}}=\pi \sqrt{\frac{m}{k}}(1+\sqrt{1+\mu})
$$

From Equation (12), the period of the ELS can be obtained:

$$
T_{E L S}=2 \pi \sqrt{\frac{m+b_{e q}}{k}}
$$

Therefore, the key is that the period of the CID system is equal to that of the ELS.

$$
T_{C I D}=T_{E L S}
$$


Combining Equation (31), Equation (32), and Equation (33), the equivalent inertance is given by

$$
b_{e q}=\left(\frac{(1+\sqrt{1+\mu})^{2}}{4}-1\right) m
$$

Finally, the equivalent inertance-mass ratio is easy to obtain, as in Wang et al. [16]:

$$
\mu_{e q}=\frac{b_{e q}}{m}=\frac{(1+\sqrt{1+\mu})^{2}}{4}-1
$$

\subsection{Equivalent Damping}

Considering one circle of free vibration, the elastic potential energy of the structure decreases due to the decay of the displacement response. During the circle, when the structure attains its peak velocity, the CID reaches its peak kinetic energy and will be separated from the whole system at the next moment. The damping of the structure also dissipates a certain amount of energy. However, the kinetic energy of the structure does not change during the circle.

For the CID system, taking the first circle in Figure 4 as an example, the system was given an initial displacement $x_{0}$ when $t=0$. The energy during the circle is balanced. That is

$$
\begin{gathered}
\Delta E_{s}=\Delta E_{k}^{C I D}+\Delta E_{d}^{s} \\
\left\{\begin{array}{c}
\Delta E_{s}=\frac{1}{2} k\left(x_{0}^{2}(t)-x_{4}^{2}(t)\right) \\
\Delta E_{C I D}=\frac{1}{2} \mu m\left(\dot{x}_{1}^{2}(t)+\dot{x}_{3}^{2}(t)\right) \\
\Delta E_{d}^{s}=\int_{0}^{t_{4}} c \dot{x}^{2}(t) d t
\end{array}\right.
\end{gathered}
$$

where $\Delta E_{s}$ represents the reduced elastic potential energy; $\Delta E_{k}^{C I D}$ is the kinetic energy obtained by the CID; $\Delta E_{d}^{s}$ is the damping energy dissipated by the structure; $x_{0}(t), x_{4}(t)$ and $\dot{x}_{1}(t), \dot{x}_{3}(t)$ represent the displacement at time $0, t_{4}$ and the velocity at time $t_{1}, t_{3}$ respectively.

For the ELS, the kinetic energy of the system also does not change during one circle. Thus, the energy equation in the ELS during a circle becomes

$$
\Delta E_{s}=\Delta E_{d}^{E L S}=\Delta E_{d}^{s}+\Delta E_{d}^{C I D}
$$

where $\Delta E_{s}$ represents the reduced elastic potential energy, and $\Delta E_{d}^{E L S}$ is the damping energy dissipated in the ELS, which consists of the damping energy dissipated by the structure, namely $\Delta E_{d}^{s}$, and the equivalent damping of the CID, namely $\Delta E_{d}^{C I D}$.

From Equations (35) and (37), the following relationship can be obtained

$$
\Delta E_{k}^{C I D}=\Delta E_{d}^{C I D}
$$

which indicates that the energy dissipated by equivalent damping $c_{e q}$ is equal to the kinetic energy obtained by the CID. Equation (38) can be expanded as

$$
\frac{1}{2} \mu m\left(\dot{x}_{1}^{2}(t)+\dot{x}_{3}^{2}(t)\right)=c_{e q} \int_{0}^{t_{4}} \dot{x}^{2}(t) d t
$$

As shown in Figure 5, the area enclosed by solid lines and the coordinate axes is equal to the area enclosed by the dotted lines and coordinate axes for a sine curve. Therefore,

$$
\int_{0}^{t_{4}} \dot{x}^{2}(t) d t=\frac{1}{2}\left(\frac{T_{C I D}}{2}\left(\dot{x}_{1}^{2}(t)+\dot{x}_{3}^{2}(t)\right)\right)
$$




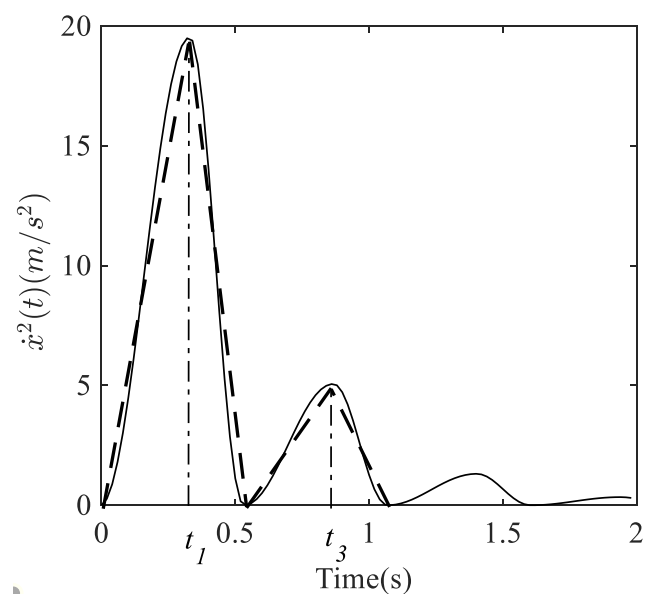

Figure 5. Square of velocity response.

Substituting Equation (40) into Equation (39), the equivalent damping $c_{e q}$ is expressed by

$$
c_{e q}=\frac{2 \mu m}{T_{C I D}}
$$

Substituting Equation (30) into Equation (41), the equivalent damping $c_{e q}$ is finally obtained:

$$
c_{e q}=\frac{2 \mu \sqrt{m k}}{\pi(1+\sqrt{1+\mu})}
$$

Substituting Equation (42) and Equation (34) into Equation (15), the equivalent damping ratio is obtained:

$$
\xi_{\text {eq }}=\frac{2 \mu}{\pi(1+\sqrt{1+\mu})^{2}}+\frac{2 \xi}{(1+\sqrt{1+\mu})}
$$

\section{ELM3 Based on SLM}

\subsection{Statistical Linearization Procedure}

In this section, the statistical linearization method (SLM) of the nonlinear SDOF system with the CID under stationary stochastic excitation is studied. The responses of the CID system are considered to have a Gaussian distribution. Firstly, Equation (9) and Equation (10) can be rewritten as

$$
\begin{gathered}
m \ddot{x}+c \dot{x}+k x+g(\dot{x}, \ddot{x})=-m \ddot{x}_{g} \\
g(\dot{x}, \ddot{x})=\mu m \ddot{x}\left(\operatorname{sgn}(\dot{x} \ddot{x})+\operatorname{sgn}^{2}(\dot{x} \ddot{x})\right) / 2
\end{gathered}
$$

where $g(\dot{x}, \ddot{x})$ is the nonlinear part of Equation (9) and $\operatorname{sgn}(\dot{x} \ddot{x})$ is the sign function.

The difference between Equation (44) and Equation (11) is

$$
\varepsilon=g(\dot{x}, \ddot{x})-b_{e q} \ddot{x}-c_{e q} \dot{x}
$$

Minimizing $E\left\{\varepsilon^{2}\right\}$, which is the expectation of $\varepsilon^{2}$, is suggested here:

$$
E\left\{\varepsilon^{2}\right\}=E\left\{\left(g(\dot{x}, \ddot{x})-b_{e q} \ddot{x}-c_{e q} \dot{x}\right)^{2}\right\}
$$


Here $b_{e q}$ and $c_{e q}$ are solved by the partial differential equation:

$$
\frac{\partial}{\partial b_{e q}} E\left\{\varepsilon^{2}\right\}=\frac{\partial}{\partial c_{e q}} E\left\{\varepsilon^{2}\right\}=0
$$

For the case that $\dot{x}(\mathrm{t})$ and $\ddot{x}(\mathrm{t})$ are Gaussian processes, this procedure finally leads to Reference [14]:

$$
\begin{aligned}
& b_{e q}=E\left\{\frac{\partial g}{\partial \ddot{x}}\right\} \\
& c_{e q}=E\left\{\frac{\partial g}{\partial \dot{x}}\right\}
\end{aligned}
$$

Substituting Equation (45) into Equation (49), $b_{e q}$ is obtained:

$$
\begin{aligned}
b_{e q} & =E\left\{\frac{\partial g}{\partial \ddot{x}}\right\} \\
& \left.=E\left\{0.5 \mu m \frac{\partial}{\partial \ddot{x}} \ddot{x}\left(\operatorname{sgn}(\dot{x} \ddot{x})+\operatorname{sgn}^{2}(\dot{x} \ddot{x})\right)\right)\right\} \\
& =0.5 \mu m E\left\{\operatorname{sgn}(\dot{x} \ddot{x})+\operatorname{sgn}^{2}(\dot{x} \ddot{x})\right\}+0.5 \mu m E\left\{\frac{\partial}{\partial \ddot{x}}\left(\operatorname{sgn}(\dot{x} \ddot{x})+\operatorname{sgn}^{2}(\dot{x} \ddot{x})\right)\right\} \\
& =0.5 \mu m E\left\{\operatorname{sgn}(\dot{x} \ddot{x})+\operatorname{sgn}^{2}(\dot{x} \ddot{x})\right\}+0.5 \mu m E\{(2 \ddot{x} \delta(\ddot{x}) \operatorname{sgn}(\dot{x}))(1+2 \operatorname{sgn}(\dot{x} \ddot{x}))\} \\
& =0.5 \mu m E\left\{\operatorname{sgn}(\dot{x} \ddot{x})+\operatorname{sgn}^{2}(\dot{x} \ddot{x})\right\}
\end{aligned}
$$

where $\delta(\ddot{x})$ is the Dirac function of $\ddot{x}$.

Here $\operatorname{sgn}(\dot{x} \ddot{x})$ is not a standard sign function due to the different time period for $\dot{x} \ddot{x}>0$ and $\ddot{x} \ddot{x}<0$. This can also be found in Figure 4. To obtain a more accurate result of Equation (51), considering the time-history of $\operatorname{sgn}(\dot{x} \ddot{x})$ in the first circle of free vibration in Figure 4:

$$
\begin{gathered}
\operatorname{sgn}(\ddot{x} \ddot{x})=\left\{\begin{array}{c}
1,0<t \leq t_{1} \\
-1, t_{1}<t \leq t_{2} \\
1, t_{2}<t \leq t_{3} \\
-1, t_{3}<t \leq t_{4}
\end{array}\right. \\
\operatorname{sgn}^{2}(\dot{x} \ddot{x})=\left\{\begin{array}{c}
0, t=0, t_{1}, t_{2}, t_{3}, t_{4} \\
1, t=\text { other time }
\end{array}\right. \\
E\{\operatorname{sgn}(\dot{x} \ddot{x})\}=\frac{2 t_{1}-t_{2}}{t_{2}}=1+\frac{2(1-\sqrt{1+\mu})}{\mu} \\
E\left\{\operatorname{sgn}^{2}(\dot{x} \ddot{x})\right\}=1
\end{gathered}
$$

in which $t_{1}$ and $t_{2}$ are obtained in Equation (26) and Equation (27), respectively. Substituting Equation (54) and Equation (55) into Equation (51), a more accurate solution of $b_{e q}$ is obtained:

$$
b_{\text {eq }}=(1+\mu-\sqrt{1+\mu}) m
$$

along with the equivalent inertance-mass ratio:

$$
\mu_{e q}=\frac{b_{e q}}{m}=(1+\mu-\sqrt{1+\mu})
$$


Substituting Equation (45) into Equation (50), $c_{e q}$ is obtained

$$
\begin{aligned}
c_{e q} & =E\left\{\frac{\partial g}{\partial \dot{x}}\right\}=E\left\{0.5 \mu m \frac{\partial}{\partial \dot{x}}\left(\ddot{x}\left(\operatorname{sgn}(\dot{x} \ddot{x})+\operatorname{sgn}^{2}(\dot{x} \ddot{x})\right)\right)\right\} \\
& =0.5 \mu m \mathrm{E}\{2 \ddot{x} \operatorname{sgn}(\ddot{x}) \delta(\dot{x})(1+2 \operatorname{sgn}(\dot{x} \ddot{x}))\} \\
& =\mu m \mathrm{E}\{|\ddot{x}| \delta(\dot{x})(1+2 \operatorname{sgn}(\dot{x} \ddot{x}))\} \\
& =\mu m \mathrm{E}\{|\ddot{x}| \delta(\dot{x})+2 \delta(\dot{x}) \operatorname{sgn}(\dot{x}) \ddot{x}\} \\
& =\mu m \mathrm{E}\{|\ddot{x}| \delta(\dot{x})\} \\
& =\mu m \iint|\ddot{x}| \delta(x) f_{\dot{x}}(\dot{x}) f_{\ddot{x}}(\ddot{x}) d \dot{x} d \ddot{x} \\
& =\frac{\mu m}{\sqrt{2 \pi} \sigma_{\dot{x}}} \int_{-\infty}^{+\infty}|\ddot{x}| f_{\ddot{x}}(\ddot{x}) d \ddot{x} \\
& =\frac{\mu m}{\sqrt{2 \pi} \sigma_{\dot{x}}} \sqrt{\frac{2}{\pi}} \sigma_{\ddot{x}}=\mu m \frac{\sigma_{\ddot{x}}}{\pi \sigma_{\dot{x}}}
\end{aligned}
$$

where $\delta(\dot{x})$ is the Dirac function of $\dot{x},|\ddot{x}|$ is the absolute value function of $\ddot{x}, f_{\dot{x}}(\dot{x})$ and $f_{\ddot{x}}(\ddot{x})$ are the probability density function of $\dot{x}$ and $\ddot{x}$, which are assumed to be independent from each other to simplify the calculation. They are all considered to be Gaussian distributions with a mean of zero. $\sigma_{\ddot{x}}$ and $\sigma_{\dot{x}}$ are the standard deviation of $\ddot{x}$ and $\dot{x}$.

\subsection{Stochastic Excitation and Response}

According to the theories of linear random vibration, the general input-output relationship for a linear SDOF system when subjected to zero-mean random excitation is that

$$
\begin{gathered}
\sigma_{x}^{2}=\int_{-\infty}^{+\infty}|H(\Omega)|^{2} S_{f}(\Omega) d \Omega \\
\sigma_{\dot{x}}^{2}=\int_{-\infty}^{+\infty}|i \Omega H(\Omega)|^{2} S_{f}(\Omega) d \Omega \\
\sigma_{\ddot{x}}^{2}=\int_{-\infty}^{+\infty}\left|\Omega^{2} H(\Omega)\right|^{2} S_{f}(\Omega) d \Omega
\end{gathered}
$$

where $\sigma_{x}^{2}, \sigma_{\dot{x}}^{2}, \sigma_{\ddot{x}}^{2}$ are the variance of the response $x, \dot{x}$ and $\ddot{x} ; H(\Omega)$ is the FRF of the linear system given by Equation (12); and $S_{f}(\Omega)$ is the auto power spectrum density of the input given by the Kanai-Tajimi model modified by Clough and Penzien [30]:

$$
\begin{gathered}
S_{f}(\Omega)=\frac{\omega_{g}^{4}+4 \xi_{g}^{2} \omega_{g}^{2} \Omega^{2}}{\left(\omega_{g}^{2}-\Omega^{2}\right)^{2}+4 \xi_{g}^{2} \omega_{g}^{2} \Omega^{2}} \frac{\Omega^{4}}{\left(\omega_{f}^{2}-\Omega^{2}\right)^{2}+4 \xi_{f}^{2} \omega_{f}^{2} \Omega^{2}} S_{0} \\
S_{0}=\frac{0.141 \xi_{g} \ddot{x}_{g 0}^{2}}{\omega_{g} \sqrt{1+4 \xi_{g}^{2}}}
\end{gathered}
$$

where $\omega_{g}$ and $\xi_{g}$ are the natural frequency and damping ratio of the soil layer, respectively; and $\omega_{f}$ and $\xi_{f}$ are the parameters of the second filter, presented in Table 1 [31]. In this paper, firm soil is considered for the calculation of equivalent parameters of the numerical study in Section $6 . S_{0}$ is the amplitude of the power spectrum of the white-noise process, which is related to the amplitude of $\ddot{x}_{g}$ [32]. It should be noted that this method could be generalized to different spectra according to the precision requirement under different excitation environments. 
Table 1. Filter parameters for different soil conditions.

\begin{tabular}{ccccc}
\hline Soil Type & $\omega_{g}(\mathrm{rad} / \mathrm{s})$ & $\xi_{g}$ & $\omega_{f}(\mathrm{rad} / \mathrm{s})$ & $\xi_{f}$ \\
\hline Firm & 15.0 & 0.6 & 1.5 & 0.6 \\
Medium & 10.0 & 0.4 & 1.0 & 0.6 \\
Soft & 5.0 & 0.2 & 0.5 & 0.6 \\
\hline
\end{tabular}

\subsection{Iterative Procedure}

The values of the equivalent linearization parameters $b_{e q}$ and $c_{e q}$ can be determined by the following iterative procedure.

Step1: Calculate $b_{e q}$ using Equation (56) and set $c_{e q}$ to 0 at the first iteration.

Step2: Calculate $\sigma_{\dot{x}}^{2}$ and $\sigma_{\ddot{x}}^{2}$ in Equations (60) and (61).

Step3: Substitute $\sigma_{\dot{x}^{\prime}}^{2}, \sigma_{\ddot{x}}^{2}$ obtained in Step 2 into Equation (58), then a new $c_{e q}$ is obtained.

Step4: Repeat Step 2 with $b_{e q}$ obtained from Equation (56) and $c_{e q}$ obtained from Step 3.

Step5: Repeat Step 3 and Step 4 several times until the value of $c_{e q}$ is accurate enough.

\section{Analysis of the Accuracy of ELMs}

In this section an analysis of the accuracy of the ELMs proposed in Section 3, Section 4, and Section 5 was conducted using a numerical study. The equivalent linearization parameters for all methods are calculated as shown in Table 2, and the parameters of the SDOF for the numerical study are set as $m=5 \mathrm{~kg}, \omega=2 \pi, \xi=0.05$.

Table 2. Equivalent linearization parameters for all methods.

\begin{tabular}{|c|c|c|c|c|c|c|c|c|c|c|c|c|}
\hline \multirow[b]{2}{*}{$\mu$} & \multicolumn{4}{|c|}{ ELM1 } & \multicolumn{4}{|c|}{ ELM2 } & \multicolumn{4}{|c|}{ ELM3 } \\
\hline & $\mu_{e q}$ & $b_{e q}$ & $c_{e q}$ & $\xi_{e q}$ & $\mu_{e q}$ & $b_{e q}$ & $c_{e q}$ & $\xi_{e q}$ & $\mu_{e q}$ & $b_{e q}$ & $c_{e q}$ & $\xi_{e q}$ \\
\hline 0.1 & 0.05 & 0.26 & 0.98 & 0.06 & 0.05 & 0.25 & 0.98 & 0.06 & 0.05 & 0.26 & 1.14 & 0.07 \\
\hline 0.2 & 0.11 & 0.53 & 1.92 & 0.08 & 0.10 & 0.49 & 1.91 & 0.08 & 0.10 & 0.52 & 2.31 & 0.08 \\
\hline 0.3 & 0.16 & 0.78 & 2.82 & 0.09 & 0.15 & 0.73 & 2.80 & 0.09 & 0.16 & 0.80 & 3.49 & 0.10 \\
\hline 0.4 & 0.21 & 1.04 & 3.69 & 0.10 & 0.19 & 0.96 & 3.66 & 0.10 & 0.22 & 1.08 & 4.69 & 0.11 \\
\hline 0.5 & 0.26 & 1.32 & 4.55 & 0.11 & 0.24 & 1.19 & 4.49 & 0.11 & 0.28 & 1.38 & 5.90 & 0.13 \\
\hline 0.6 & 0.32 & 1.58 & 5.37 & 0.12 & 0.28 & 1.41 & 5.30 & 0.12 & 0.34 & 1.68 & 7.12 & 0.14 \\
\hline 0.7 & 0.37 & 1.83 & 6.16 & 0.13 & 0.33 & 1.63 & 6.08 & 0.13 & 0.40 & 1.98 & 8.35 & 0.15 \\
\hline 0.8 & 0.41 & 2.06 & 6.91 & 0.13 & 0.37 & 1.85 & 6.83 & 0.14 & 0.46 & 2.29 & 9.58 & 0.17 \\
\hline 0.9 & 0.47 & 2.37 & 7.70 & 0.14 & 0.41 & 2.07 & 7.57 & 0.14 & 0.52 & 2.61 & 10.81 & 0.18 \\
\hline 1.0 & 0.53 & 2.63 & 8.46 & 0.15 & 0.46 & 2.29 & 8.28 & 0.15 & 0.59 & 2.93 & 12.05 & 0.19 \\
\hline
\end{tabular}

Figure 6 shows the results of the equivalent linearization parameters intuitively. As can be seen, the equivalent inertance-mass ratio is almost linearly related to the inertance-mass ratio, and so is the equivalent damping ratio; the difference is that the equivalent damping ratio obtained from ELM3 is larger than that from ELM1 and ELM2. 


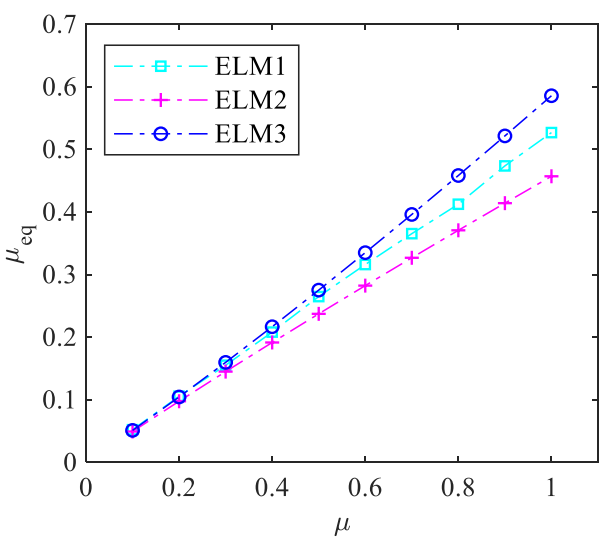

(a)

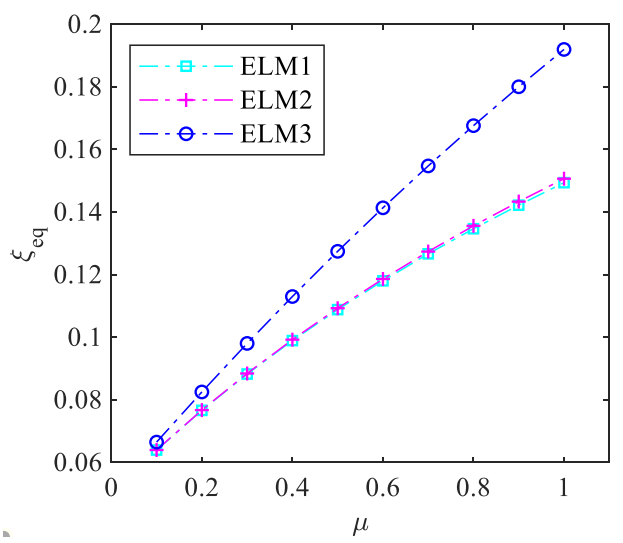

(b)

Figure 6. Equivalent inertance-mass ratio and equivalent damping ratio varying with inertance-mass ratio: (a) Equivalent inertance-mass ratio; (b) Equivalent damping ratio.

\subsection{Response of Free Vibration}

Free vibration is a motion that can reflect the inherent characteristics of the structure, including natural frequency and damping. Figure 7a shows the displacement response of the CID system and the ELS with different ELMs; it seems that ELM2 based on period and energy is the most accurate solution followed by ELM2, and ELM3 has the maximum error, as seen in Figure 7b.

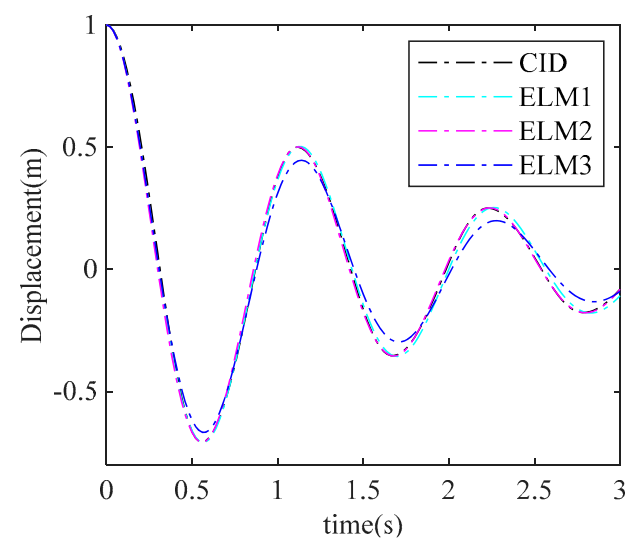

(a)

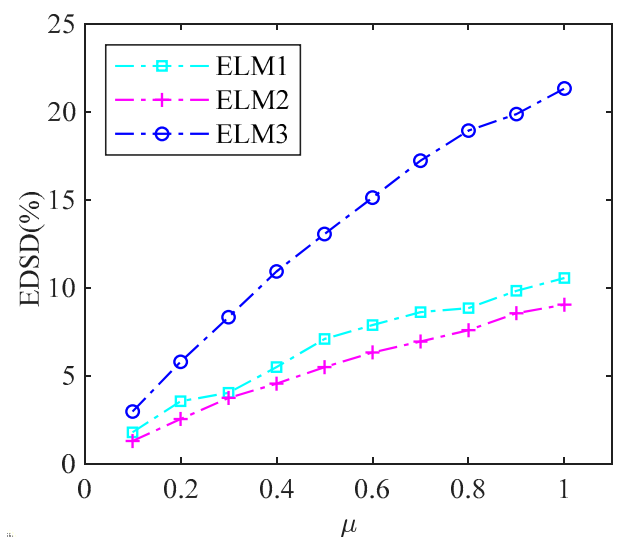

(b)

Figure 7. Displacement response of free vibration and its error analysis for all equivalent linearization methods (ELMs). (a) Displacement response when $\mu$ is 0.5 ; (b) Error of displacement standard deviation.

The error of displacement standard deviation (EDSD) is calculated with Equation (64):

$$
E D S D=\frac{\operatorname{std}\left(x_{E L S}-x_{C I D}\right)}{s t d\left(x_{C I D}\right)} \times 100 \%
$$

where $x_{C I D}$ and $x_{E L S}$ are the displacement responses of the CID system and ELS with different ELMs, respectively, and $\operatorname{std}(\cdot)$ is the operation of standard deviation.

\subsection{Response to Harmonic Excitations}

Figure 8 shows the comparison of the steady-state peak response of displacement between the CID system and the ELS with different ELMs under harmonic excitations $\sin (\Omega \mathrm{t})$. As shown in Figure $8 \mathrm{~b}$, ELM1 is the most closed approach to the CID system followed by ELM2, and ELM3 still seems to be the method with the biggest error. 


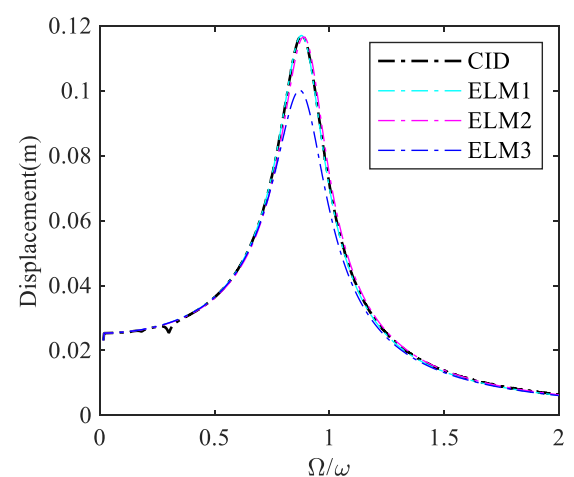

(a)

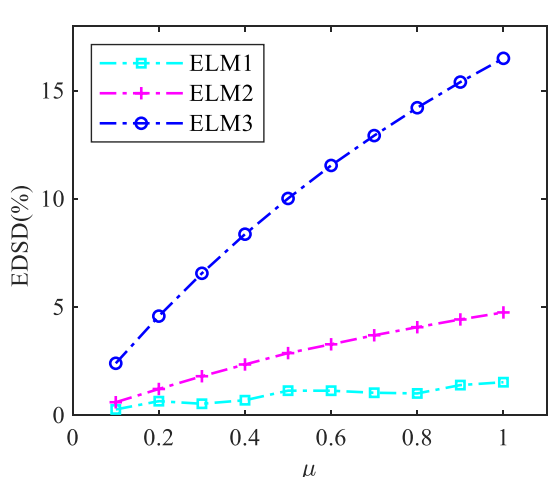

(b)

Figure 8. Displacement response to harmonic excitations and its error analysis for all ELMs. (a)

Displacement response when $\mu$ is 0.5 ; (b) Error of displacement standard deviation varying with $\mu$.

\subsection{Response to Seismic Excitations}

Considering the analysis in Sections 6.1 and 6.2, it can be concluded that ELM1 and ELM2 perform better in the comparison of displacement standard deviation. Since ELM1 and ELM2 are based on the adaption of FRF and the decay of free vibration, the comparison seems unfair for ELM3. In this section, three natural seismic waves and an artificial wave of firm soil are selected for time-history analysis of the CID system and ELS. The peak ground acceleration (PGA) of the seismic waves are all adjusted by $0.2 \mathrm{~g}$, with $\mathrm{g}$ being the value of gravity acceleration. Figure 9 shows the displacement time-history curves of the CID system and ELS with different ELMs when the inertance-mass ration is 0.5. The results show that ELM3 seems to be the most accurate method, although sometimes the response of ELM3 is slightly smaller than the CID system. The responses of ELM1 and ELM2 are commonly slightly larger than the CID system. The error analysis in Figure 10 shows that the error of ELM1 and ELM2 are quite the same. The response of the CID system shows a high demand for damping, since the equivalent damping obtained with ELM3 is larger than with ELM1 and ELM2.

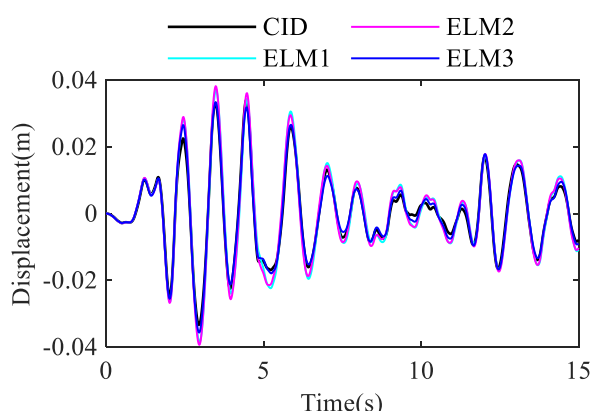

(a)

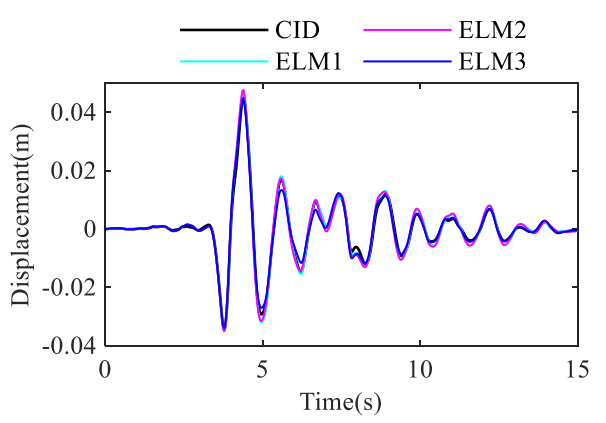

(c)

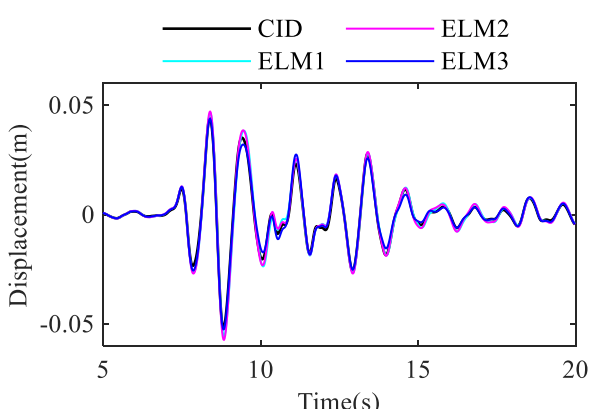

(b)

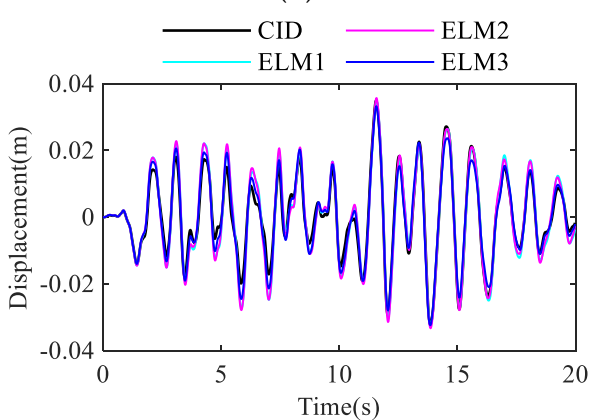

(d)

Figure 9. Displacement response of the CID system and equivalent linear system (ELS) to seismic excitations when $\mu$ is 0.5. (a) Elcentro wave; (b) Kobe wave; (c) Northridge wave; (d) Artifical wave. 


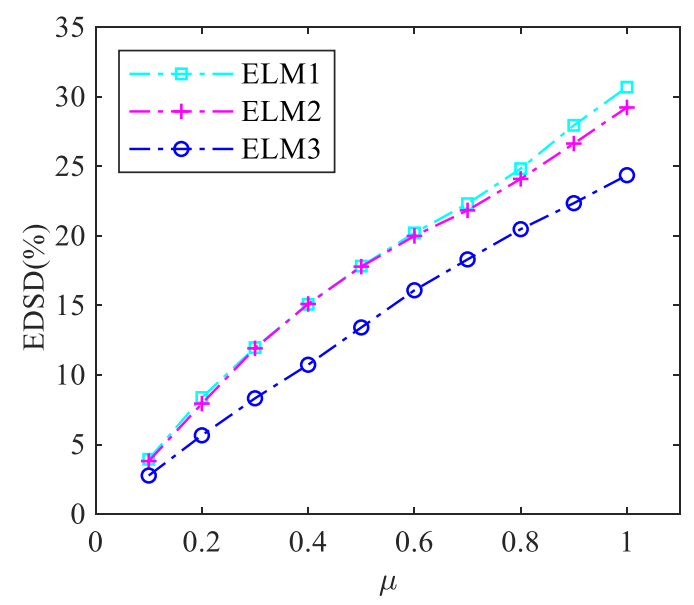

Figure 10. Mean error of displacement standard deviation for all ELMs to four seismic waves varying with $\mu$.

\section{Conclusions}

The equivalent linearization methods for a system with a clutching inerter damper are developed in this study. First, the equations of motion for the system with IMD and CID are built using mechanical analysis. The simulation results show the superiority of the CID system. Subsequently, three ELMs are proposed based on different principles, which can be applied using the derived formulas or procedures. Finally, a numerical study is conducted to verify the feasibility of different ELMs.

All the ELMs proposed in this paper are proved to have enough accuracy for small inertance-mass ratios, with the error being within an acceptable range. As the inertance-mass ratio increases, the error also increases for all methods. Responses to free vibration and harmonic excitations show the accuracy of ELM1 and ELM2 in describing the inherent properties of the structure. However, it seems that the CID system will show a high demand for damping under seismic excitations and ELM3 will be more accurate. From another point of view, ELM1 and ELM2 will always yield larger results than the CID system, compared with ELM3, which is a more secure or conservative choice for response estimation and structure design. Since ELM2 has a clear physical meaning and direct formulas, it should be a recommended method for small inertance-mass ratios.

On the one hand, the CID system retains the mass amplification effect of the IMD system, which is effective in mitigating the intensity of the input and extending the structural vibration period. On the other hand, the equivalent damping effect of the CID system reduces the sensitivity of the system to the frequency of input and further mitigates the response. The ELS accurately reflects the characteristics of the CID system and can evaluate the response of the nonlinear system with acceptable error. The feasibility of the ELS can be used for the performance design of the CID system.

Author Contributions: All authors made scientific contributions: L.L. conceived the idea of this research, Q.L. made the calculations and wrote the manuscript, and H.Q. revised the manuscript.

Funding: This work was supported in part by the National Natural Science Foundation of China under Award No. 51678116.

Conflicts of Interest: The authors declare no conflict of interest.

\section{References}

1. Li, L.; Song, G.B.; Ou, J.P. DNN Based Fault Tolerant Control of Nonlinear Structural Vibration with Actuator Faults. Adv. Struct. Eng. 2011, 14, 871-879. [CrossRef]

2. Huo, L.S.; Song, G.B.; Nagarajaiah, S.; Li, H.N. Semi-active vibration suppression of a space truss structure using a fault tolerant controller. J. Vib. Control 2012, 18, 1436-1453. [CrossRef]

3. Lin, C.J.; Lee, C.Y.; Liu, Y. Vibration Control Design for a Plate Structure with Electrorheological ATVA Using Interval Type-2 Fuzzy System. Appl. Sci. 2017, 7, 707. [CrossRef] 
4. Li, C.; Zhang, T.Y.; Zhou, S.T.; Xiao, Y.Q.; Hu, G. Passive Vibration Control of a Semi-Submersible Floating Offshore Wind Turbine. Appl. Sci. 2017, 7, 509. [CrossRef]

5. Song, G.B.; Cai, C.S.; Li, H.N. Energy Dissipation and Vibration Control: Modeling, Algorithm, and Devices. Appl. Sci. 2017, 7, 801. [CrossRef]

6. Zhang, Z.; Ou, J.P.; Li, D.S.; Zhang, S.F. Optimization Design of Coupling Beam Metal Damper in Shear Wall Structures. Appl. Sci. 2017, 7, 137. [CrossRef]

7. Qian, H.; Li, H.; Song, G.B. Experimental investigations of building structure with a superelastic shape memory alloy friction damper subject to seismic loads. Smart Mater. Struct. 2016, 25, 125026. [CrossRef]

8. Liu, M.; Zhou, P.; Li, H. Novel Self-Centering Negative Stiffness Damper Based on Combination of Shape Memory Alloy and Prepressed Springs. J. Aerosp. Eng. 2018, 31, 04018100. [CrossRef]

9. Lin, W.; Song, G.B.; Chen, S.H. PTMD Control on a Benchmark TV Tower under Earthquake and Wind Load Excitations. Appl. Sci. 2017, 7, 425. [CrossRef]

10. Zhao, N.; Lu, C.W.; Chen, M.Y.; Luo, N.; Liu, C.Q. Parametric Study of Pounding Tuned Mass Damper Based on Experiment of Vibration Control of a Traffic Signal Structure. J. Aerosp. Eng. 2018, 31, 04018108. [CrossRef]

11. Wang, H.; Li, L.Y.; Song, G.B.; Dabney, J.B.; Harman, T.L. A new approach to deal with sensor errors in structural controls with MR damper. Smart Struct. Syst. 2015, 16, 329-345. [CrossRef]

12. Li, L.Y.; Liang, H.Z. Semi-active Control of Structural Nonlinear Vibration Considering the MR Damper Model. J. Aerosp. Eng. 2018, 31, 04018095-1. [CrossRef]

13. Smith, M.C. Synthesis of mechanical networks: The inerter. IEEE Trans. Autom. Control 2002, 47, 1648-1662. [CrossRef]

14. Chen, M.Z.Q.; Papageorgiou, C.; Scheibe, F.; Wang, F.C.; Smith, M.C. The missing mechanical circuit element. IEEE Circuits Syst. Mag. 2009, 9, 10-26. [CrossRef]

15. Makris, N.; Kampas, G. Seismic Protection of Structures with Supplemental Rotational Inertia. J. Eng. Mech. 2016, 142, 04016089. [CrossRef]

16. Wang, M.; Sun, F. Displacement reduction effect and simplified evaluation method for SDOF systems using a clutching inerter damper. Earthq. Eng. Struct. Dyn. 2018, 47, 1651-1672. [CrossRef]

17. Rosenblueth, E.; Herrera, I. On a kind of hysteretic damping. J. Eng. Mech. Div. 1964, 90, 37-48.

18. Iwan, W.D. Estimating Earthquake Response of Simple Hysteretic Structures. J. Eng. Mech. Div. ASCE 1979, 3, 391-405.

19. Iwan, W.D. Estimating inelastic response spectra from elastic spectra. Earthq. Eng. Struct. Dyn. 1980, 8, 375-388. [CrossRef]

20. Hwang, J.S. Evaluation of Equivalent Linear Analysis Methods of Bridge Isolation. J. Struct. Eng. 1996, 122, 972-976. [CrossRef]

21. Iwan, W.D.; Yang, I.M. Application of Statistical Linearization Techniques to Nonlinear Multi-degree-of-Freedom Systems. J. Appl. Mech. 1972, 39, 545. [CrossRef]

22. Atalik, T.S.; Utku, S. Stochastic linearization of multi-degree-of-freedom non-linear systems. Earthq. Eng. Struct. Dyn. 2010, 4, 411-420. [CrossRef]

23. Roberts, J.B.; Spanos, P.D. Random Vibration and Statistical Linearization; Wiley: Chichester, UK, 2003.

24. Hurtado, J.E.; Barbat, A.H. Equivalent linearization of the Bouc-Wen hysteretic model. Eng. Struct. 2000, 22, 1121-1132. [CrossRef]

25. Fujimura, K.; Kiureghian, A.D. Tail-equivalent linearization method for nonlinear random vibration. Probabilistic Eng. Mech. 2007, 22, 63-76. [CrossRef]

26. Broccardo, M.; Kiureghian, A.D. Multicomponent Nonlinear Stochastic Dynamic Analysis by Tail-Equivalent Linearization. J. Eng. Mech. 2016, 142, 04015100. [CrossRef]

27. Wang, Z.; Kiureghian, A.D. Tail-Equivalent Linearization of Inelastic Multi-support Structures Subjected to Spatially Varying Stochastic Ground Motion. J. Eng. Mech. 2016, 142, 04016053. [CrossRef]

28. Wang, Z.; Song, J. Equivalent linearization method using Gaussian mixture (GM-ELM) for nonlinear random vibration analysis. Struct. Saf. 2016, 64, 9-19. [CrossRef]

29. Nakamura, Y.; Fukukita, A.; Tamura, K.; Yamazaki, I.; Matsuoka, T.; Hiramoto, K.; Sunakoda, K. Seismic response control using electromagnetic inertial mass dampers. Earthq. Eng. Struct. Dyn. 2014, 43, 507-527. [CrossRef]

30. Clough, R.W.; Penzien, J. Dynamics of structures; Computers and Structures Inc.: Berkeley, CA, USA, 2003. 
31. Kiureghian, A.D.; Neuenhofer, A. Response spectrum method for multi-support seismic excitations. Earthq. Eng. Struct. Dyn. 1992, 21, 713-740. [CrossRef]

32. Buchholdt, H.A. Structural Dynamics for Engineers; Earthquake Resistant Structures; Thomas Teldfort: London, UK, 1997. 